\title{
Efeito de Diferentes Períodos de Controle das Plantas Daninhas na Produtividade da Cultura da Cebola ${ }^{1}$
}

\author{
Effect of Different Weed Control Periods on Onion Crop Yield \\ SOARES, D.J. ${ }^{2}$, GRAVENA, R. ${ }^{3}$ e PITELLI, R.A. ${ }^{4}$
}

\begin{abstract}
RESUMO - Foi realizado um experimento em Monte Alto-SP, visando estudar os efeitos de diferentes períodos de controle das plantas daninhas sobre a produção de bulbos da cultura da cebola (Allium cepa), cultivar 'Mercedes', no sistema de transplantio. O delineamento experimental utilizado foi o de blocos ao acaso, com quatro repetições. Parte dos tratamentos experimentais foi disposta num esquema fatorial $4 \times 6$, em que constituíram variáveis quatro periodos em que se fez o controle (0-0, 0-7, 0-14 e 0-21 DAT) e seis períodos em que se reiniciou o controle das plantas daninhas prolongando até a colheita: $28,42,56,70,84$ e 98 DAT). Duas testemunhas foram adotadas: uma com controle e outra sem controle das plantas daninhas durante todo o ciclo da cultura. Lycopersicon esculentum, Portulaca oleracea, Eragrostis pilosa e Galinsoga parviflora foram as plantas daninhas mais importantes na área. Não houve interação entre os diferentes periodos de controle das plantas daninhas. O controle inicial destas plantas deve se prolongar até 14 DAT e ser reiniciado aos 28 DAT, a fim de prevenir reduções significativas na produtividade em relação à testemunha no limpo A convivência com as plantas daninhas durante todo o ciclo da cebola reduziu a produtividade em $94,5 \%$.
\end{abstract}

Palavras-chave: Allium cepa, comunidade infestante, competição.

\begin{abstract}
An experiment was carried out in Monte Alto, SP to study the effects of different of weed control periods on the yield of onion (Allium cepa) bulb, 'Mercedes' cultivar, under the transplanting system. The experiment was arranged in a completely randomized block design, with four replications. The treatments were arranged in $4 \times 6$ factorial design with four initial weed removal periods (after transplanting until 0, 7, 14 and 21 days) and six final weed removal periods (from 28, 42, 56, 70, 84 or 98 days after transplanting until harvest). Two controls were adapted with and without weed control throughout the onion cycle. Lycopersicon esculentum, Portulaca oleracea, Eragrostis pilosa and Galinsoga parviflora were the most important weeds in the area. No interaction occurred between the different weed controls. Weed control should be maintained until 14 DAT and be restarted at 28 DAT to prevent significant yield reductions in relation to the control without weeding. Weed interference throughout the onion cycle reduced yield in $94.5 \%$.
\end{abstract}

Key words: Allium cepa, weed community, competition.

\footnotetext{
Recebido para publicação em 8.1.2004 e na forma revisada em 17.12.2004.

2 Eng.-Agr., Mestrando em Produção Vegetal pela Faculdade de Ciências Agrárias e Veterinárias da Universidade Estadual Paulista - FCAV/UNESP; 14884-900 Jaboticabal-SP, Bolsista FAPESP. ${ }^{3}$ Eng.-Agr., Doutorando em Fitotecnia pela Escola Superior de Agricultura "Luiz de Queiroz" - ESALQ/USP, 13418-900 Piracicaba-SP. ${ }^{4}$ Professor Titular do Departamento de Biologia Aplicada à Agropecuária-FCAV/UNESP.
} 


\section{INTRODUÇÃO}

Parte dos estudos científicos sobre interferência das plantas daninhas em culturas agrícolas visa determinar os períodos ou as épocas que são críticas na interação culturamato. No entanto, poucos trabalhos avaliam os aspectos fitossociológicos no desenvolvimento da comunidade infestante e seus reflexos no crescimento e na produção da cultura. No Brasil, esses estudos são escassos e restritos às culturas de grande interesse econômico, como o milho, a soja e o arroz inundado. Há, portanto, uma grande carência de trabalhos cientificos dessa natureza envolvendo culturas de hortaliças (Pitelli, 1987).

Parece evidente que um pequeno número de espécies predomina numericamente nas comunidades infestantes em áreas de olericultura. Esse fato está ligado às linhas evolutivas desenvolvidas pelas espécies presentes, principalmente em termos de estratégia reprodutiva (Pitelli, 1987). Segundo Grime (1979), as plantas ruderais habitam locais de baixo estresse ambiental e alta intensidade de distúrbio, como são as áreas de olericultura, bastante irrigadas e adubadas, mas intensivamente cultivadas com espécies de ciclo relativamente curto. Essas espécies são usualmente herbáceas, com rápido ciclo de desenvolvimento e alta produção de propágulos e se caracterizam por alta agressividade e baixa capacidade competitiva.

Essas plantas ruderais, nas áreas de plantio de cebola, encontram condições plenas para exercer seu potencial de interferência, pois a cultura apresenta baixa capacidade competitiva (Heemst, 1985). A presença das plantas daninhas ocasiona importantes alterações na planta da cebola; como conseqüência final, a produtividade desta cultura decresce drasticamente, podendo atingir até $100 \%$ de perdas na produção de bulbos comercializáveis. Alguns valores de redução na produtividade encontrados na literatura variam de 36 a 96\% (Paller et al., 1971; Deuber \& Forster, 1975; Bond \& Burston, 1996; Soares et al., 2003).

O presente trabalho objetivou estudar a produtividade da cebola transplantada, em função de períodos de controle das plantas daninhas estabelecidos em diferentes estádios da cultura, visando localizar a época em que o controle das plantas daninhas é necessário para prevenir perdas na produtividade da cultura.

\section{MATERIAL E MÉTODOS}

O experimento foi conduzido no período de abril a agosto de 2002, no município de Monte Alto-SP, em solo Podzólico Vermelho-Amarelo $\mathrm{Tb}$, classe textural arenosa. O solo apresentou $\mathrm{pH}\left(\mathrm{CaCl}_{2}\right)$ de 5,$9 ; 13$ g.dm ${ }^{-3}$ de matéria orgânica; teores de $\mathrm{P}=330 \mathrm{mg} \mathrm{dm}^{-3} ; \mathrm{K}^{+}=5,3$; $\mathrm{Ca}^{+2}=48 ; \mathrm{Mg}^{+2}=10, \mathrm{H}+\mathrm{Al}=13 ; \mathrm{SB}=63,3$ (expressos em mmol $\mathrm{dm}^{-3}$ ); e $\mathrm{V} \%=83$. A adubação de plantio foi feita com $500 \mathrm{~kg} \mathrm{ha}^{-1}$ da fórmula comercial 04-14-08 e $50 \mathrm{~kg} \mathrm{ha}^{-1} \mathrm{de}$ termofosfato Yoorin. Durante o mês de abril de 2002, foi realizada a semeadura do cultivar Mercedes em canteiros para produção de mudas, sendo o transplantio destas para o campo realizado aos 35 dias após a semeadura, utilizando o espaçamento de 0,3 m entre linhas e $0,08 \mathrm{~m}$ entre plantas, totalizando 41,7 plantas $\mathrm{m}^{-2}$. O delineamento experimental utilizado foi o de blocos ao acaso, com quatro repetições. As parcelas experimentais foram constituídas de quatro linhas de plantio com 2,0 m de comprimento, apresentando uma área total de $2,4 \mathrm{~m}^{2}$. Foram consideradas como área útil as duas linhas centrais, desprezando-se 0,3 m de cada extremidade. Os tratamentos foram dispostos num esquema fatorial 4 × $6+2$, em que constituíram variáveis quatro periodos iniciais de controle: 0-0, 0-7, 0-14 e 0-21 dias após o transplantio (DAT) e seis épocas para o reinício do controle das plantas daninhas $(28,42,56,70,84$ e 98 DAT), mais duas testemunhas (uma com e outra sem controle de plantas daninhas durante todo o ciclo da cultura). Como exemplo, o primeiro tratamento teve controle inicial do mato no dia do transplantio e permaneceu sem controle até os 28 DAT, sendo o controle reiniciado aos 28 DAT e mantido até a colheita. O tratamento 8 teve um controle inicial até os 7 DAT e permaneceu sem o controle até os 42 DAT, sendo este reiniciado aos 42 DAT e mantido até a colheita. O controle de plantas daninhas foi feito por meio de capinas manuais.

A comunidade infestante foi avaliada ao final de cada período de convivência, sendo coletadas todas as plantas daninhas presentes em 0,5 $\mathrm{m}^{2}$ da área útil de cada parcela, 
correspondendo a duas subamostras de $0,25 \mathrm{~m}^{2}$, retiradas ao acaso.

As espécies de plantas daninhas foram identificadas, quantificadas e levadas ao laboratório, onde foram lavadas e depois secas em estufa com circulação forçada de ar a 70 $80^{\circ} \mathrm{C}$, por 96 horas, e pesadas em balança de precisão de 0,01 g. Apenas foram consideradas as partes aéreas das plantas daninhas.

$\mathrm{Na}$ comunidade infestante, foi realizado um estudo fitossociológico das populações presentes, conforme metodologia proposta por Mueller-Dombois \& Ellemberg (1974). Foram determinadas, para cada espécie, a densidade relativa (porcentagem de indivíduos de uma espécie em relação ao total de indivíduos da comunidade); a constância relativa (porcentagem de amostras em que os individuos de uma espécie foram detectados em relação ao número total de amostras efetuadas - a constância relativa é a relação percentual da constância de uma espécie em relação à soma das constâncias de todas as espécies da comunidade); a dominância relativa (porcentagem de biomassa seca acumulada por uma espécie em relação à biomassa seca da comunidade); e a importância relativa (porcentagem do valor de importância de uma espécie, obtida da soma dos três valores anteriormente citados, em relação ao valor de importância total da comunidade).

$\mathrm{Na}$ comunidade infestante, foram ainda realizados estudos de regressão para acúmulo de biomassa seca e densidade de plantas daninhas ao final dos períodos de convivência e foi determinado o índice de diversidade de Shannon - Weaver $(\mathrm{H})$, citado por Pinto-Coelho (2000). Esse índice é calculado por meio da seguinte fórmula:

$$
H=\sum_{i=1}^{S} p_{i} \ln p_{i}
$$

em que s é o número de espécies e $p_{i}$ é a proporção da amostra contendo indivíduos da espécie $i$.

A colheita da cebola foi realizada aos 105 dias após o transplantio. Os bulbos foram contados, classificados, de acordo com o diâmetro transversal, em classe 5 (diâmetro maior que $9 \mathrm{~cm}$ ), classe 4 (diâmetro entre 7 e $9 \mathrm{~cm}$ ), classe 3 (diâmetro entre 7 e $5 \mathrm{~cm}$ ), classe 2 (diâmetro entre 5 e 3,5 cm) e refugo (diâmetro menor que $3,5 \mathrm{~cm}$ ), conforme classificação adotada pela Ceagesp (1999), e pesados em balança eletrônica com precisão de 0,01 g. Em seguida, foram calculadas as distribuições percentuais em cada classe de tamanho.

Os dados de produção total de bulbos foram submetidos à análise de variância pelo teste F. As médias foram confrontadas pelo teste de comparação múltipla de Tukey em nível de 5\% de probabilidade. Para análise de tendência dos efeitos dos períodos de interferência sobre a produção, foram realizados estudos de regressão, segundo modelo sigmoidal de Boltzmann, que obedece à seguinte equação: $\mathrm{y}=\mathrm{A}_{2}+\left[\left(\mathrm{A}_{1}-\mathrm{A}_{2}\right) /\left[1-\exp \left(\mathrm{x}-\mathrm{x}_{0}\right) / \mathrm{dx}\right]\right.$, em que y é a produtividade da cebola, em porcentagem; $\mathrm{x}$ é o limite superior do periodo de convivência; $A_{1}$ e $A_{2}$ são as assintotas da curva; $x_{0}$ é o limite superior do período de convivência, que corresponde ao ponto de inflexão da curva; e dx é o parâmetro que indica velocidade de perda ou ganho de produção ( $\operatorname{tg} \alpha$ no ponto $\mathrm{x}_{0}$ ).

\section{RESULTADOS E DISCUSSÃO}

\section{Comunidade infestante}

As plantas daninhas que ocorreram na área experimental foram carrapicho-de-carneiro (Acanthospermum hispidum, ACNHI), caruru (Amaranthus hybridus, AMAHY), capimmarmelada (Brachiaria plantaginea, BRAPL), tiririca (Cyperus rotundus, CYPRO), capimcolchão (Digitaria horizontalis, DIGHO), capimpé-de-galinha (Eleusine indica, ELEIN), capimbarbicha-de-alemão (Eragrostis pilosa, ERAPI), picão-branco (Galinsoga parviflora, GASPA), mentruz (Lepidium virginicum, LEPVI), tomate (Lycopersicon esculentum) e beldroega (Portulaca oleracea, POROL). A maior parte das espécies presentes pode ser considerada ruderal, de acordo com os critérios de Grime (1979). O tomate rasteiro, cultivado no ano anterior, apresentava um elevado banco de sementes no solo, germinando alguns indivíduos na área experimental, sendo considerado neste trabalho como planta daninha, levando em conta a definição de planta daninha como uma planta que ocorre onde não é desejada e que, de algum modo, promove prejuízos às atividades do homem (Pitelli, 1985).

Planta Daninha, Viçosa-MG, v. 22, n. 4, p. 517-527, 2004 
Na Figura 1-A está apresentada a marcha de acúmulo de matéria seca pela comunidade infestante na cultura da cebola, em função dos períodos iniciais de controle. O modelo de regressão adotado foi o de Gaussian, e os respectivos parâmetros se encontram na Tabela 1. O acúmulo máximo observado esteve na faixa entre 350 e $400 \mathrm{~g} \mathrm{~m}^{-2}$, que ocorreu aos 56 dias após o transplantio, no grupo de tratamentos em que o período inicial de controle das plantas daninhas foi de 0-0 DAT. Nos outros grupos de tratamento, os maiores acúmulos observados estiveram na faixa entre 150 e $200 \mathrm{~g} \mathrm{~m}^{-2}$, ocorrendo aos 84 dias. Esses resultados sugerem que um controle inicial da comunidade infestante é importante para reduzir sua capacidade de acúmulo de matéria seca e de mobilização de recursos do meio e, portanto, seu poder de interferência competitiva sobre a cultura da cebola.

As curvas de densidade de plantas da comunidade infestante (Figura 1-B) obedeceram às tendências sigmoidais e os respectivos parâmetros se encontram na Tabela 1. Houve tendência de redução do número de plantas daninhas na comunidade infestante com o tempo, sendo mais evidente no grupo de tratamentos em que o período inicial de controle foi de 0-0 DAT. Isso pode ser explicado pelo fato de espécies como Portulaca oleraceae terem sua população drasticamente reduzida em virtude do aumento e da competição de outras populações de plantas de ciclo mais tardio.

Na Tabela 2 estão apresentados os resultados referentes ao estudo fitossociológico desenvolvido com a comunidade infestante no grupo de tratamentos em que o periodo inicial de controle das plantas daninhas foi de 0-0 DAT, nas diferentes épocas de avaliação durante o ciclo da cebola. Os dados referentes à constância relativa não serão discutidos, em razão do pequeno tamanho da área experimental e, relativamente, do pequeno número de amostras.

Até os 56 dias, três populações apresentaram maiores importâncias relativas, sendo elas $P$. oleracea, L. esculentum e E. pilosa, devido principalmente à densidade relativa, no caso de $P$. oleracea e E. pilosa, e dominância relativa, em se tratando de $L$. esculentum (Tabela 2). Aos 70 dias, o L. esculentum se apresentou como a mais importante $(36,82 \%$ de
I.R.), seguida de BRAPL, que teve a segunda maior participação $(14,13 \%)$ em termos de dominância relativa, e de E. pilosa, que se destacou pela sua densidade relativa $(13,20 \%)$ (Tabela 2). Nas duas últimas avaliações (84 e 98 dias), o L. esculentum manteve sua maior importância relativa, tendo como principal componente dessa importância a sua dominância relativa, que chegou a 82,21\% aos 98 dias (Tabela 2). Em seguida, destacaram-se G. parviflora, com 26,17\% aos 84 dias e $12,22 \%$ aos 98 dias, e E. pilosa, com 10,5 e $11,14 \%$, respectivamente (Tabela 2).
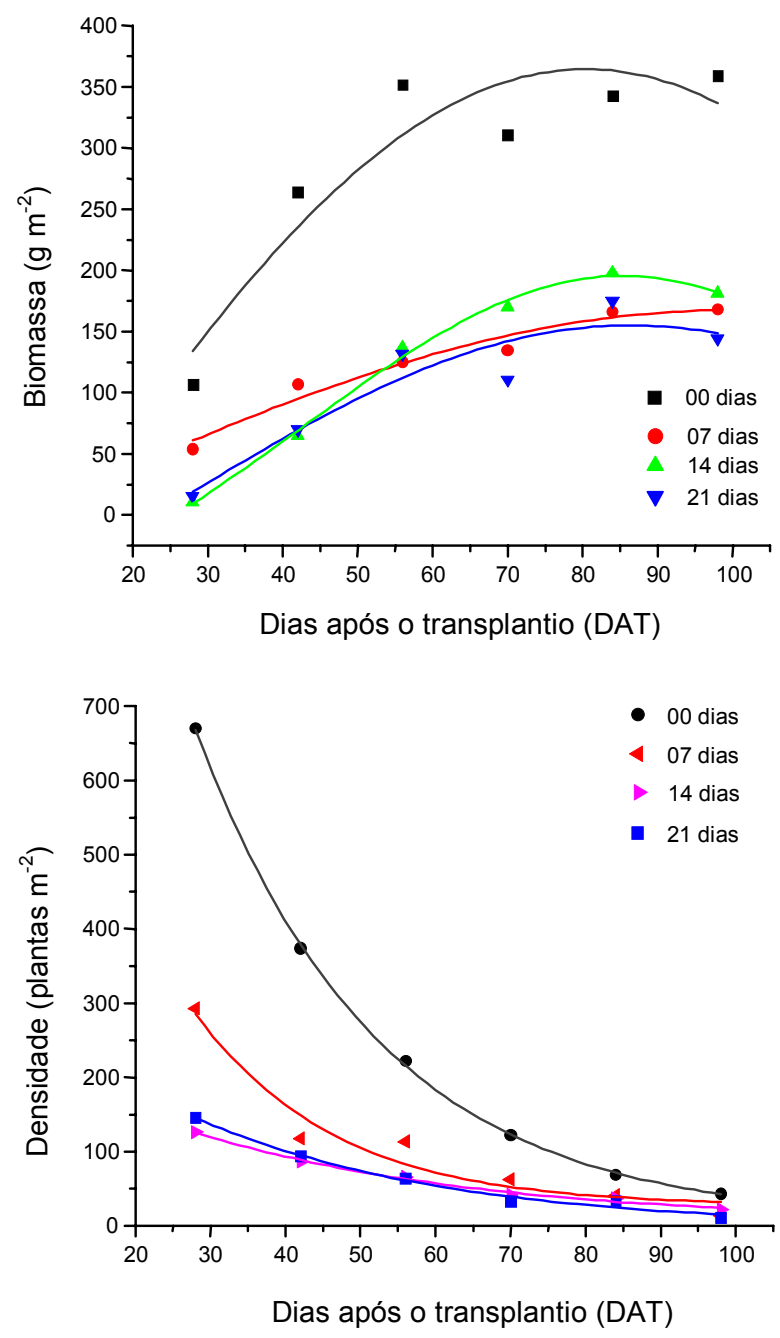

Figura 1 - Variação temporal dos valores de biomassa seca acumulada (A) e densidade de indivíduos (B) da comunidade infestante na cultura da cebola cultivar Mercedes, após os respectivos períodos iniciais de controle. FCAV/UNESP, Jaboticabal-SP, 2003. 
Tabela 1 - Parâmetros obtidos pelo modelo de Gaussian aplicado aos acúmulos de biomassa seca pelas plantas daninhas e parâmetros obtidos pelo modelo de Boltzmann aplicado à densidade de plantas da comunidade infestante nos diferentes períodos iniciais de controle na cultura da cebola 'Mercedes'. FCAV/UNESP. Jaboticabal-SP, 2003

\begin{tabular}{|c|c|c|c|c|c|}
\hline \multirow{2}{*}{$\begin{array}{l}\text { Período inicial } \\
\text { de controle }\end{array}$} & \multicolumn{5}{|c|}{ Gaussian } \\
\hline & $\mathrm{R}^{2}$ & y0 & $\mathrm{xc}$ & $\mathrm{w}$ & A \\
\hline $0-0 \mathrm{DAT}^{1 /}$ & 0,8672 & $-1017,79$ & 80,44 & 173,70 & 300912,97 \\
\hline 0-7 DAT & 0,9595 & $-261,70$ & 100,32 & 191,34 & 103004,75 \\
\hline $0-14$ DAT & 0,9954 & $-115,84$ & 85,31 & 84,93 & 33140,98 \\
\hline $0-21$ DAT & 0,8908 & $-238,19$ & 86,39 & 126,64 & 62430,60 \\
\hline \multirow{2}{*}{$\begin{array}{c}\text { Período inicial } \\
\text { de controle }\end{array}$} & \multicolumn{5}{|c|}{ Boltzmann } \\
\hline & $\mathrm{R}^{2}$ & A1 & A2 & $\mathrm{x} 0$ & $\mathrm{dx}$ \\
\hline $0-0$ DAT & 0,9997 & 32297,96 & 5,89 & $-64,79$ & 24,00 \\
\hline $0-7$ DAT & 0,9520 & 3239,97 & 26,89 & $-14,75$ & 17,54 \\
\hline $0-14$ DAT & 0,9938 & 3280,67 & 5,64 & $-92,45$ & 36,83 \\
\hline $0-21$ DAT & 0,9879 & 2005,22 & $-1,10$ & $-50,97$ & 31,08 \\
\hline
\end{tabular}

${ }^{1} \mathrm{DAT}=$ Dias após o transplantio.
Na Tabela 3 estão apresentados os índices fitossociológicos para as populações da comunidade infestante em que o período inicial de controle foi de 0-7 DAT. Nessa comunidade, a população de $L$. esculentum se apresentou como a mais importante em todas as épocas de avaliação, com exceção da primeira (28 dias), com valores de I.R. variando entre $13,86 \%$ e $67,55 \%$, aos 28 e 98 dias, respectivamente (Tabela 3) - isso por causa de sua grande participação em termos de dominância relativa. As outras espécies mais importantes depois do L. esculentum nessa comunidade foram P. oleracea, com $36,17 \%$ de I.R. aos 28 dias; E. pilosa, com $20,37 \%$ de I.R. aos 56 dias; G. parviflora, com $21,18 \%$ e $20,75 \%$ de I.R. aos 70 e 84 dias, respectivamente; e A. hybridus, com 12,37\% de I.R. aos 98 dias (Tabela 3).

No grupo de tratamentos em que o período inicial de controle foi de 0-14 DAT, a população

Tabela 2 - Valores dos índices fitossociológicos de densidade (De.R.), constância (Co.R.), dominância (Do.R.) e importância (I.R.) relativas das populações componentes da comunidade infestante no grupo de tratamentos em que o período inicial de controle foi de 0 dia, na cultura da cebola, nas diferentes épocas de avaliação. FCAV/UNESP, Jaboticabal-SP, 2003

\begin{tabular}{|c|c|c|c|c|c|c|c|c|c|c|c|c|}
\hline \multirow{2}{*}{ Espécie } & De.R. & Co.R. & Do.R. & I.R. & De.R. & Co.R. & Do.R. & I.R. & De.R. & Co.R. & Do.R. & I.R. \\
\hline & \multicolumn{4}{|c|}{$28 \mathrm{DAT}^{1 /}$} & \multicolumn{4}{|c|}{$42 \mathrm{DAT}$} & \multicolumn{4}{|c|}{$56 \mathrm{DAT}$} \\
\hline CYPRO & 5,38 & 9,76 & 3,73 & 6,29 & 11,54 & 11,76 & 1,98 & 8,43 & 8,83 & 7,89 & 1,75 & 6,16 \\
\hline DIGHO & 1,07 & 9,76 & 1,31 & 4,05 & 0,30 & 2,94 & 0,13 & 1,12 & 1,43 & 7,89 & 8,72 & 6,02 \\
\hline TOMATE & 13,46 & 9,76 & 15,68 & 12,97 & 20,99 & 11,76 & 45,29 & 26,01 & 36,99 & 10,53 & 68,72 & 38,75 \\
\hline ERAPI & 16,23 & 9,76 & 2,37 & 9,45 & 20,39 & 11,76 & 17,52 & 16,56 & 19,57 & 10,53 & 4,50 & 11,53 \\
\hline POROL & 42,12 & 7,32 & 64,43 & 37,96 & 17,99 & 11,76 & 10,66 & 13,47 & 8,35 & 10,53 & 2,30 & 7,06 \\
\hline AMAHY & 5,30 & 7,32 & 0,52 & 4,38 & 10,94 & 11,76 & 2,12 & 8,28 & 7,16 & 7,89 & 6,27 & 7,11 \\
\hline LEPVI & 2,06 & 9,76 & 0,43 & 4,08 & 4,50 & 5,88 & 0,81 & 3,73 & 2,15 & 10,53 & 0,18 & 4,29 \\
\hline BRAPL & 3,72 & 9,76 & 6,84 & 6,77 & 6,00 & 8,82 & 8,97 & 7,93 & 2,39 & 10,53 & 3,48 & 5,46 \\
\hline ELEIN & 3,56 & 9,76 & 1,13 & 4,82 & 0,90 & 5,88 & 0,72 & 2,50 & 6,44 & 7,89 & 0,42 & 4,92 \\
\hline GASPA & 5,98 & 9,76 & 2,46 & 6,06 & 5,25 & 11,76 & 11,17 & 9,39 & 5,97 & 10,53 & 3,12 & 6,54 \\
\hline \multirow[t]{2}{*}{ ACNHI } & 1,11 & 7,32 & 1,10 & 3,17 & 1,20 & 5,88 & 0,63 & 2,57 & 0,72 & 5,26 & 0,53 & 2,17 \\
\hline & \multicolumn{4}{|c|}{70 DAT } & \multicolumn{4}{|c|}{$84 \mathrm{DAT}$} & \multicolumn{4}{|c|}{98 DAT } \\
\hline CYPRO & 8,45 & 12,35 & 0,83 & 7,21 & 0,76 & 5,00 & 0,05 & 1,94 & 0,00 & 0,00 & 0,00 & 0,00 \\
\hline DIGHO & 0,00 & 0,00 & 0,00 & 0,00 & 0,00 & 0,00 & 0,00 & 0,00 & 0,00 & 0,00 & 0,00 & 0,00 \\
\hline TOMATE & 36,29 & 12,35 & 61,81 & 36,82 & 41,67 & 20,00 & 54,10 & 38,59 & 53,73 & 26,67 & 82,21 & 54,20 \\
\hline ERAPI & 13,20 & 12,35 & 5,54 & 10,36 & 9,09 & 15,00 & 7,41 & 10,50 & 16,42 & 13,33 & 3,68 & 11,14 \\
\hline POROL & 11,55 & 9,26 & 2,10 & 7,63 & 0,00 & 0,00 & 0,00 & 0,00 & 2,99 & 6,67 & 1,44 & 3,70 \\
\hline AMAHY & 4,12 & 9,26 & 2,23 & 5,21 & 4,55 & 15,00 & 1,40 & 6,98 & 5,97 & 13,33 & 4,69 & 8,00 \\
\hline LEPVI & 2,89 & 6,17 & 0,53 & 3,20 & 2,27 & 5,00 & 0,27 & 2,51 & 0,00 & 0,00 & 0,00 & 0,00 \\
\hline BRAPL & 11,55 & 10,49 & 14,13 & 12,06 & 8,33 & 15,00 & 4,11 & 9,15 & 8,96 & 13,33 & 1,56 & $\begin{array}{l}7,95 \\
7,95\end{array}$ \\
\hline ELEIN & 4,12 & 9,26 & 0,18 & 4,52 & 6,82 & 5,00 & 0,65 & 4,16 & 0,00 & 0,00 & 0,00 & 0,00 \\
\hline GASPA & 4,95 & 12,35 & 10,06 & 9,12 & 26,52 & 20,00 & 32,01 & 26,17 & 10,45 & 20,00 & 6,22 & 12,22 \\
\hline ACNHI & 2,89 & 6,17 & 2,58 & 3,88 & 0,00 & 0,00 & 0,00 & 0,00 & 1,49 & 6,67 & 0,20 & 2,79 \\
\hline
\end{tabular}

1/ DAT - Dias após o transplantio. 
Tabela 3 - Valores dos índices fitossociológicos de densidade (De.R.), constância (Co.R.), dominância (Do.R.) e importância (I.R.) relativas das populações componentes da comunidade infestante no grupo de tratamentos em que o período inicial de controle foi de 7 dias após o transplantio, na cultura da cebola, nas diferentes épocas de avaliação. FCAV/UNESP, Jaboticabal-SP, 2003

\begin{tabular}{|c|c|c|c|c|c|c|c|c|c|c|c|c|}
\hline \multirow{2}{*}{ Espécie } & De.R. & Co.R. & Do.R. & I.R. & De.R. & Co.R. & Do.R. & I.R. & De.R. & Co.R. & Do.R. & I.R. \\
\hline & \multicolumn{4}{|c|}{$28 \mathrm{DAT}^{1 /}$} & \multicolumn{4}{|c|}{42 DAT } & \multicolumn{4}{|c|}{56 DAT } \\
\hline CYPRO & 2,10 & 10,00 & 1,45 & 4,52 & 6,53 & 8,57 & 5,77 & 6,96 & 7,30 & 6,45 & 0,41 & 4,72 \\
\hline DIGHO & 2,61 & 10,00 & 4,16 & 5,59 & 1,34 & 5,71 & 0,67 & 2,58 & 0,41 & 3,23 & 0,38 & 1,34 \\
\hline TOMATE & 12,95 & 10,00 & 18,63 & 13,86 & 28,82 & 11,43 & 59,85 & 33,37 & 18,05 & 12,90 & 44,18 & 25,04 \\
\hline ERAPI & 18,59 & 10,00 & 3,60 & 10,73 & 10,18 & 8,57 & 6,20 & 8,32 & 28,40 & 12,90 & 19,80 & 20,37 \\
\hline POROL & 35,66 & 10,00 & 62,84 & 36,17 & 11,72 & 11,43 & 5,00 & 9,38 & 13,18 & 12,90 & 4,55 & 10,21 \\
\hline AMAHY & 14,47 & 10,00 & 1,93 & 8,80 & 15,95 & 11,43 & 2,90 & 10,09 & 15,01 & 12,90 & 6,20 & 11,37 \\
\hline LEPVI & 2,69 & 7,50 & 0,34 & 3,51 & 2,50 & 11,43 & 1,19 & 5,04 & 3,25 & 3,23 & 0,50 & 2,32 \\
\hline BRAPL & 1,85 & 7,50 & 3,05 & 4,13 & 7,20 & 8,57 & 1,11 & 5,63 & 0,20 & 3,23 & 1,33 & 1,59 \\
\hline ELEIN & 3,03 & 10,00 & 2,14 & 5,06 & 1,92 & 5,71 & 0,80 & 2,81 & 2,03 & 9,68 & 0,62 & 4,11 \\
\hline GASPA & 3,87 & 10,00 & 1,63 & 5,17 & 12,49 & 8,57 & 15,33 & 12,13 & 10,75 & 12,90 & 18,76 & 14,14 \\
\hline \multirow[t]{2}{*}{$\mathrm{ACNHI}$} & 2,19 & 5,00 & 0,22 & 2,47 & 1,34 & 8,57 & 1,18 & 3,70 & 1,42 & 9,68 & 3,28 & 4,79 \\
\hline & \multicolumn{4}{|c|}{70 DAT } & \multicolumn{4}{|c|}{84 DAT } & \multicolumn{4}{|c|}{$98 \mathrm{DAT}$} \\
\hline CYPRO & 8,03 & $\begin{array}{l}9,09 \\
9,09\end{array}$ & 1,64 & 6,26 & 3,06 & 4,17 & 0,28 & 2,50 & 0,00 & 0,00 & 0,00 & 0,00 \\
\hline DIGHO & 0,00 & 0,00 & 0,00 & 0,00 & 0,00 & 0,00 & 0,00 & 0,00 & 0,00 & 0,00 & 0,00 & 0,00 \\
\hline TOMATE & 18,07 & 12,12 & 36,27 & 22,15 & 26,91 & 16,67 & 45,38 & 29,65 & 77,48 & 36,36 & 88,79 & 67,55 \\
\hline ERAPI & 30,12 & 12,12 & 13,86 & 18,70 & 8,56 & 12,50 & 13,16 & 11,41 & 8,11 & 27,27 & 0,13 & 11,84 \\
\hline POROL & 14,46 & 9,09 & 2,31 & 8,62 & 6,73 & 4,17 & 0,69 & 3,86 & 0,00 & 0,00 & 0,00 & 0,00 \\
\hline AMAHY & 7,23 & 12,12 & 4,39 & 7,91 & 25,69 & 12,50 & 4,29 & 14,16 & 9,01 & 27,27 & 0,84 & 12,37 \\
\hline LEPVI & 0,80 & 6,06 & 0,13 & 2,33 & 4,28 & 12,50 & 4,30 & 7,03 & 0,00 & 0,00 & 0,00 & 0,00 \\
\hline BRAPL & 2,81 & 9,09 & 4,08 & 5,33 & 2,45 & 8,33 & 5,85 & 5,54 & 5,41 & 9,09 & 10,24 & 8,25 \\
\hline ELEIN & 1,20 & 6,06 & 0,17 & 2,48 & 0,31 & 4,17 & 0,00 & 1,49 & 0,00 & 0,00 & 0,00 & 0,00 \\
\hline GASPA & 15,26 & 12,12 & 36,17 & 21,18 & 20,18 & 16,67 & 25,39 & 20,75 & 0,00 & 0,00 & 0,00 & 0,00 \\
\hline ACNHI & 2,01 & 12,12 & 0,98 & 5,04 & 1,83 & 8,33 & 0,66 & 3,61 & 0,00 & 0,00 & 0,00 & 0,00 \\
\hline
\end{tabular}

1/ DAT - Dias após o transplantio.

de L. esculentum novamente foi a mais importante em épocas de avaliação, com exceção dos 42 dias. Os índices apresentados na Tabela 4 mostram que, depois do L. esculentum, $P$. oleracea se destacou na primeira e terceira épocas (28 e 56 dias), devido principalmente à sua densidade relativa: $25,62 \%$ e $27,17 \%$, respectivamente. A partir dos 70 dias, assumiram maior importância relativa - depois do L. esculentum - as espécies E. pilosa e G. parviflora. A primeira, por causa de sua representatividade numérica (De.R entre 16,12 e 44,10\%), e a segunda devido à maior participação em termos de biomassa acumulada (Do.R entre 10,62 e 26,72\%) (Tabela 4).

Por fim, no grupo de tratamentos em que o período inicial de controle foi de 0-21 DAT (Tabela 5), a população de L. esculentum apresentou o mesmo padrão de comportamento das comunidades comentadas anteriormente.
Foram obtidos maiores valores de I.R (entre 11,86 e $59,02 \%$ ) em todas as épocas de avaliação, com exceção da primeira (28 dias). Quanto às outras populações, nota-se que $P$. oleracea teve destaque aos 28, 42 e 56 dias, principalmente pelos índices de De.R. apresentados; E. pilosa se destacou também aos 28 , 42, 56 e 70 dias pelos altos índices de De.R. e de Do.R. aos 84 dias. Aos 70 e 84 dias, G. parviflora ficou ao lado de E. pilosa, em termos de importância relativa, por ter apresentado altos valores de Do.R (17,20 e 20,21\%, respectivamente) (Tabela 5). Na última avaliação, realizada aos 98 dias, G. parviflora foi a população mais importante depois de L. esculentum, seguida de $A$. hybridus, também por ter apresentado maior indice de Do.R $(18,27 \%)$ em relação a esse último (Tabela 5).

Em suma, considerando o comportamento das populações mais importantes nas quatro 
comunidades infestantes avaliadas (quatro grupos de tratamentos), pode-se dizer que a população de $L$. esculentum se destacou por apresentar uma grande representatividade numérica, acompanhada de grande acúmulo de biomassa seca, em relação às outras populações, independentemente da época em que se iniciou seu desenvolvimento. Em segundo lugar destacou-se E. pilosa, pelo fato de apresentar maiores índices de De.R na maioria das épocas de avaliação. Isso fez com que se tornasse a segunda população mais importante nas comunidades avaliadas. $P$. oleracea e G. parviflora se destacaram como as espécies mais importantes depois de $L$. esculentum e E. pilosa, porém em épocas diferentes de avaliação. Nas primeiras avaliações $(28,42$ e 56 dias), P. oleracea se destacou, tendo como principal componente de sua importância relativa a densidade relativa, enquanto
G. parviflora se destacou a partir dos 70 dias, devido principalmente aos seus índices de Do.R.

O indice de diversidade de ShannonWeaver, citado por Pinto-Coelho (2000), considera o número de populações presentes e a participação percentual de cada uma na comunidade. Neste estudo, o indice foi calculado considerando-se as participações das populações em termos de densidade relativa (De.R.), dominância relativa (Do.R) e importância relativa (I.R.) (Figura 2).

O indice de Shannon-Weaver é máximo quando todas as populações têm a mesma participação na comunidade, ou seja, a equitabilidade das populações é máxima. Para as quatro comunidades em análise (0-0, 0-7, 0-14 e 0-21 DAT), o valor máximo teórico decresceu de maneira mais acentuada a partir dos

Tabela 4 - Valores dos índices fitossociológicos de densidade (De.R.), constância (Co.R.), dominância (Do.R.) e importância (I.R.) relativas das populações componentes da comunidade infestante no grupo de tratamentos em que o período inicial de controle foi de 14 dias após o transplantio, na cultura da cebola, nas diferentes épocas de avaliação. FCAV/UNESP, Jaboticabal-SP, 2003

\begin{tabular}{|c|c|c|c|c|c|c|c|c|c|c|c|c|}
\hline \multirow{2}{*}{ Espécie } & De.R. & Co.R. & Do.R. & I.R. & De.R. & Co.R. & Do.R. & I.R. & De.R. & Co.R. & Do.R. & I.R. \\
\hline & \multicolumn{4}{|c|}{$28 \mathrm{DAT}^{1 /}$} & \multicolumn{4}{|c|}{$42 \mathrm{DAT}$} & \multicolumn{4}{|c|}{$56 \mathrm{DAT}$} \\
\hline CYPRO & 10,36 & 10,00 & 16,01 & 12,12 & 5,68 & 9,52 & 2,61 & 5,94 & 4,53 & 5,71 & 0,24 & 3,49 \\
\hline DIGHO & 4,63 & 7,50 & 25,25 & 12,46 & 1,35 & 7,14 & 1,04 & 3,18 & 1,89 & 5,71 & 0,13 & 2,58 \\
\hline TOMATE & 22,02 & 10,00 & 21,48 & 17,83 & 12,18 & 9,52 & 22,30 & 14,67 & 18,11 & 11,43 & 42,30 & 23,95 \\
\hline ERAPI & 16,10 & 10,00 & 3,03 & 9,71 & 45,47 & 9,52 & 30,03 & 28,34 & 20,75 & 11,43 & 5,51 & 12,56 \\
\hline POROL & 25,62 & 10,00 & 13,95 & 16,52 & 10,28 & 9,52 & 9,24 & 9,68 & 27,17 & 11,43 & 16,59 & 18,40 \\
\hline AMAHY & 9,99 & 10,00 & 3,89 & 7,96 & 8,93 & 9,52 & 2,81 & 7,09 & 8,68 & 11,43 & 2,97 & 7,69 \\
\hline LEPVI & 1,85 & 7,50 & 1,11 & 3,49 & 1,35 & 7,14 & 0,34 & 2,95 & 1,51 & 5,71 & 0,13 & 2,45 \\
\hline BRAPL & 2,41 & 10,00 & 10,59 & 7,67 & 2,57 & 9,52 & 11,19 & 7,76 & 0,94 & 8,57 & 1,95 & 3,82 \\
\hline ELEIN & 2,59 & 10,00 & 1,73 & 4,77 & 4,60 & 9,52 & 3,93 & 6,02 & 1,51 & 5,71 & 0,43 & 2,55 \\
\hline GASPA & 2,96 & 7,50 & 1,37 & 3,94 & 5,41 & 9,52 & 11,97 & 8,97 & 12,26 & 11,43 & 27,72 & 17,14 \\
\hline \multirow[t]{2}{*}{ ACNHI } & 1,48 & 7,50 & 1,59 & 3,52 & 2,17 & 9,52 & 4,56 & 5,42 & 2,64 & 11,43 & 2,03 & 5,37 \\
\hline & \multicolumn{4}{|c|}{70 DAT } & \multicolumn{4}{|c|}{84 DAT } & \multicolumn{4}{|c|}{98 DAT } \\
\hline CYPRO & 11,24 & 11,43 & 3,64 & 8,77 & 14,47 & 10,00 & 0,31 & 8,26 & 0,00 & 0,00 & 0,00 & 0,00 \\
\hline DIGHO & 0,00 & 0,00 & 0,00 & 0,00 & 0,00 & 0,00 & 0,00 & 0,00 & 0,00 & 0,00 & 0,00 & 0,00 \\
\hline TOMATE & 11,80 & 11,43 & 36,70 & 19,97 & 23,03 & 13,33 & 44,90 & 27,09 & 28,72 & 22,22 & 70,58 & 40,51 \\
\hline ERAPI & 22,47 & 11,43 & 18,61 & 17,50 & 16,12 & 13,33 & 11,96 & 13,80 & 44,10 & 16,67 & 13,13 & 24,63 \\
\hline POROL & 16,85 & 11,43 & 3,75 & 10,68 & 8,55 & 3,33 & 0,33 & 4,07 & 0,00 & 0,00 & 0,00 & 0,00 \\
\hline AMAHY & 14,04 & 11,43 & 4,04 & 9,84 & 5,26 & 6,67 & 1,76 & 4,56 & 5,64 & 16,67 & 2,99 & 8,43 \\
\hline LEPVI & 1,12 & 5,71 & 0,97 & 2,60 & 2,63 & 10,00 & 0,39 & 4,34 & 0,00 & 0,00 & 0,00 & 0,00 \\
\hline BRAPL & 2,81 & 5,71 & 1,69 & 3,41 & 8,55 & 13,33 & 19,13 & 13,67 & 4,10 & 16,67 & 2,18 & 7,65 \\
\hline ELEIN & 4,49 & 11,43 & 1,36 & 5,76 & 1,97 & 6,67 & 0,09 & 2,91 & 0,00 & 0,00 & 0,00 & 0,00 \\
\hline GASPA & 12,36 & 11,43 & 26,72 & 16,84 & 16,45 & 13,33 & 18,27 & 16,02 & 15,38 & 22,22 & 10,62 & 16,08 \\
\hline $\mathrm{ACNHI}$ & 2,81 & 8,57 & 2,52 & 4,63 & 2,96 & 10,00 & 2,86 & 5,27 & 2,05 & 5,56 & 0,50 & 2,70 \\
\hline
\end{tabular}

1/ DAT - Dias após o transplantio. 
Tabela 5 - Valores dos índices fitossociológicos de densidade (De.R.), constância (Co.R.), dominância (Do.R.) e importância (I.R.) relativas das populações componentes da comunidade infestante no grupo de tratamentos em que o período inicial de controle foi de 21 dias após o transplantio, na cultura da cebola, nas diferentes épocas de avaliação. FCAV/UNESP, Jaboticabal-SP, 2003

\begin{tabular}{|c|c|c|c|c|c|c|c|c|c|c|c|c|}
\hline \multirow{2}{*}{ Espécie } & De.R. & Co.R. & Do.R. & I.R. & De.R. & Co.R. & Do.R. & I.R. & De.R. & Co.R. & Do.R. & I.R. \\
\hline & \multicolumn{4}{|c|}{$28 \mathrm{DAT}^{1 /}$} & \multicolumn{4}{|c|}{42 DAT } & \multicolumn{4}{|c|}{56 DAT } \\
\hline CYPRO & 10,96 & 10,81 & 6,25 & 9,34 & 9,85 & 10,53 & 2,99 & 7,79 & 7,48 & 9,38 & 0,85 & 5,90 \\
\hline DIGHO & 1,35 & 8,11 & 3,04 & 4,17 & 1,23 & 7,89 & 0,49 & 3,21 & 0,79 & 6,25 & 0,09 & 2,37 \\
\hline TOMATE & 9,78 & 10,81 & 14,99 & 11,86 & 18,47 & 10,53 & 24,28 & 17,76 & 18,11 & 12,50 & 64,73 & 31,78 \\
\hline ERAPI & 14,84 & 10,81 & 7,99 & 11,21 & 18,72 & 10,53 & 17,49 & 15,58 & 22,44 & 12,50 & 10,23 & 15,06 \\
\hline POROL & 43,34 & 10,81 & 58,16 & 37,44 & 26,60 & 10,53 & 14,53 & 17,22 & 25,98 & 12,50 & 5,03 & 14,50 \\
\hline AMAHY & 9,95 & 10,81 & 2,53 & 7,76 & 8,62 & 10,53 & 5,54 & 8,23 & 11,02 & 12,50 & 4,40 & 9,31 \\
\hline LEPVI & 0,34 & 2,70 & 0,61 & 1,21 & 0,99 & 7,89 & 0,25 & 3,04 & 2,76 & 6,25 & 0,55 & 3,19 \\
\hline BRAPL & 1,01 & 5,41 & 1,59 & 2,67 & 5,17 & 10,53 & 15,93 & 10,54 & 2,36 & 9,38 & 4,67 & 5,47 \\
\hline ELEIN & 1,52 & 8,11 & 0,47 & 3,36 & 0,25 & 2,63 & 0,09 & 0,99 & 0,39 & 3,13 & 0,16 & 1,22 \\
\hline GASPA & 5,06 & 10,81 & 1,30 & 5,72 & 8,37 & 10,53 & 15,75 & 11,55 & 7,48 & 9,38 & 8,78 & 8,54 \\
\hline \multirow[t]{2}{*}{ ACNHI } & 1,85 & 10,81 & 3,09 & 5,25 & 1,72 & 7,89 & 2,66 & 4,09 & 1,18 & 6,25 & 0,51 & 2,65 \\
\hline & \multicolumn{4}{|c|}{70 DAT } & \multicolumn{4}{|c|}{84 DAT } & \multicolumn{4}{|c|}{98 DAT } \\
\hline CYPRO & 16,55 & 14,29 & 3,90 & 11,58 & 0,00 & 0,00 & 0,00 & 0,00 & 0,00 & 0,00 & 0,00 & 0,00 \\
\hline DIGHO & 0,00 & 0,00 & 0,00 & 0,00 & 0,00 & 0,00 & 0,00 & 0,00 & 0,00 & 0,00 & 0,00 & 0,00 \\
\hline TOMATE & 28,97 & 14,29 & 54,94 & 32,73 & 32,03 & 20,00 & 45,15 & 32,39 & 62,22 & 36,36 & 78,48 & 59,02 \\
\hline ERAPI & 15,86 & 10,71 & 10,19 & 12,25 & 22,06 & 20,00 & 25,36 & 22,47 & 0,00 & 0,00 & 0,00 & 0,00 \\
\hline POROL & 11,03 & 10,71 & 0,64 & 7,46 & 22,06 & 10,00 & 1,27 & 11,11 & 0,00 & 0,00 & 0,00 & 0,00 \\
\hline AMAHY & 11,03 & 10,71 & 6,84 & 9,53 & 7,83 & 20,00 & 4,56 & 10,80 & 15,56 & 18,18 & 8,65 & 14,13 \\
\hline LEPVI & 0,69 & 3,57 & 0,33 & 1,53 & 0,00 & 0,00 & 0,00 & 0,00 & 4,44 & 9,09 & 0,14 & 4,56 \\
\hline BRAPL & 5,52 & 10,71 & 4,70 & 6,98 & 3,56 & 5,00 & 3,03 & 3,86 & 0,00 & 0,00 & 0,00 & 0,00 \\
\hline ELEIN & 2,07 & 3,57 & 0,18 & 1,94 & 0,00 & 0,00 & 0,00 & 0,00 & 0,00 & 0,00 & 0,00 & 0,00 \\
\hline GASPA & 6,90 & 14,29 & 17,20 & 12,79 & 11,39 & 15,00 & 20,21 & 15,53 & 15,56 & 27,27 & 11,99 & 18,27 \\
\hline ACNHI & 1,38 & 7,14 & 1,08 & 3,20 & 1,07 & 10,00 & 0,43 & 3,83 & 2,22 & 9,09 & 0,75 & 4,02 \\
\hline
\end{tabular}

1/ DAT - Dias após o transplantio.

70 dias, com a redução do número de populações sobreviventes. Houve maior estabilidade no indice de diversidade quando se consideraram a participação numérica e a importância relativa das populações.

\section{Produção da cebola}

Na Tabela 6 encontram-se os valores médios da produção total de bulbos de cebola, considerando as variáveis principais e os resultados das análises de variância correspondentes. Para essa variável (períodos iniciais e de reinício de controle) não foi estatisticamente detectada qualquer interação entre os periodos. Assim, apenas foram comparadas as médias dentro de cada período inicial ou de reinício de controle das plantas daninhas. As maiores produções foram obtidas quando os periodos iniciais de controle foram de 0-14 e 0-21 DAT e o controle reiniciado aos 28 DAT. Embora o grupo de tratamentos em que o controle foi de 0-0 DAT tenha apresentado semelhança estatística aos dois maiores periodos iniciais de controle (0-14 e 0-21 DAT), a perda na produção foi pronunciada para o produtor.

Apenas houve interação entre os tratamentos fatoriais e as testemunhas estudadas; a testemunha mantida livre da presença das plantas daninhas apresentou produção semelhante à obtida pelos tratamentos que reiniciaram o controle aos 28 DAT. O teste de Tukey, apesar de não ser o mais adequado para tratamentos quantitativos, é útil para separar 


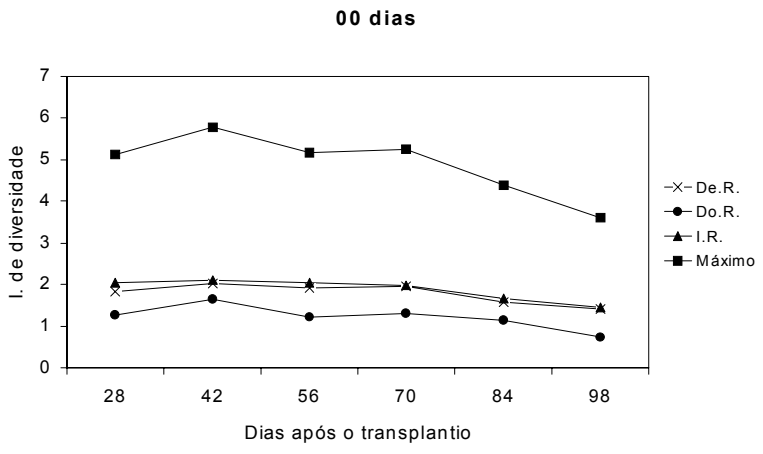

07 dias

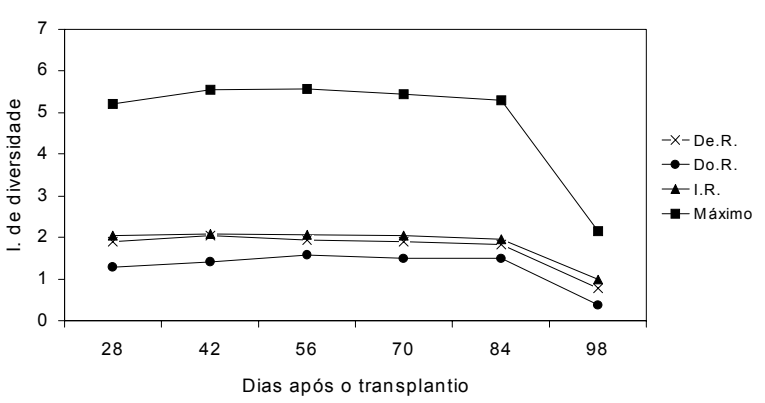

14 dias

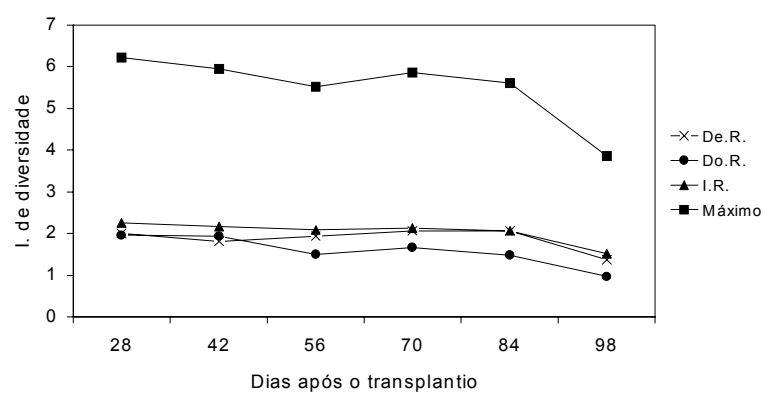

21 dias

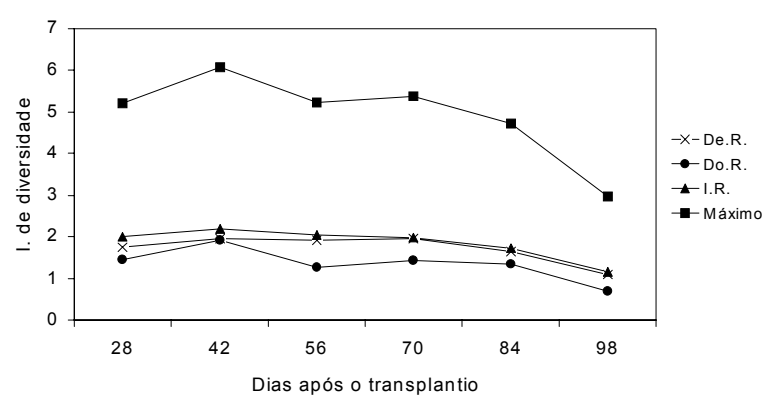

Figura 2 - Valores do índice de diversidade de Shannon-Weaver das comunidades infestantes nos grupos de tratamentos com diferentes períodos iniciais de controle das plantas daninhas (0-0, 0-7, 0-14 e 0-21 dias após o transplantio), nas diferentes épocas de avaliação, considerando a densidade relativa (De.R.), a dominância relativa (De.R.), a importância relativa (I.R.) e o valor máximo das populações de plantas daninhas. FCAV/UNESP, Jaboticabal-SP, 2003. o período de convivência que reduz significativamente o componente da produção, quando comparado com a testemunha no limpo. Nesse sentido, a produção total de bulbos teve redução significativa quando o controle das plantas daninhas se reiniciou aos 42 DAT, independentemente do periodo inicial de controle. Esse resultado sugere que o controle inicial das plantas daninhas deve se prolongar até 14 DAT e ser reiniciado aos 28 DAT, a fim de prevenir reduções significativas na produtividade em relação à testemunha no limpo. A convivência com as plantas daninhas durante todo o ciclo da cultura reduziu a produtividade da cebola em $94,5 \%$.

Tabela 6 - Produção total de bulbos submetidos a diferentes períodos iniciais e períodos com reinício de controle das plantas daninhas na cultura da cebola transplantada, cultivar Mercedes. FCAV-UNESP. Jaboticabal-SP, 2003

\begin{tabular}{|c|c|}
\hline Variável principal & \multirow{2}{*}{$\begin{array}{l}\text { Produção total } \\
\quad\left(\mathrm{kg} \mathrm{m}^{-2}\right)\end{array}$} \\
\hline Período inicial de controle (pi) & \\
\hline $00-00 \mathrm{DAT}^{1 /}$ & $2,04 \mathrm{ab}^{2 /}$ \\
\hline 00-07 DAT & $1,88 \mathrm{~b}$ \\
\hline $00-14 \mathrm{DAT}$ & $2,57 \mathrm{a}$ \\
\hline $00-21 \mathrm{DAT}$ & $2,52 \mathrm{a}$ \\
\hline \multicolumn{2}{|c|}{ Período em que se reiniciou o controle (pr) } \\
\hline 28 DAT-colheita ${ }^{3 /}$ & $6,46 \mathrm{a}$ \\
\hline 42 DAT-colheita & $4,47 \mathrm{~b}$ \\
\hline 56 DAT-colheita & $1,44 \mathrm{c}$ \\
\hline 70 DAT-colheita & $0,63 \mathrm{~d}$ \\
\hline 84 DAT-colheita & $0,41 \mathrm{~d}$ \\
\hline 98 DAT-colheita & $0,11 \mathrm{~d}$ \\
\hline \multicolumn{2}{|l|}{ Testemunhas $(\mathrm{t})$} \\
\hline $\mathrm{T}_{1}$ - Limpo & $5,88 \mathrm{a}$ \\
\hline $\mathrm{T}_{2}-$ Sujo & $0,33 \mathrm{~b}$ \\
\hline \multicolumn{2}{|l|}{ Análise de variância } \\
\hline $\mathrm{F}_{(\mathrm{pi})}$ & $4,77 * *$ \\
\hline$F_{(p r)}$ & $182,32 * *$ \\
\hline$F_{(t)}$ & $103,49^{* *}$ \\
\hline$F_{(t \text { vs fatorial })}$ & $8,95^{* *}$ \\
\hline$F_{(\text {pix pr) }}$ & $1,42 \mathrm{~ns}$ \\
\hline $\operatorname{DMS}_{(\mathrm{pi})}$ & $0,5862 \mathrm{~kg} \mathrm{~m}^{-2}$ \\
\hline $\mathrm{DMS}_{(\mathrm{pr})}$ & $0,7983 \mathrm{~kg} \mathrm{~m}^{-2}$ \\
\hline $\mathrm{DMS}_{(\mathrm{t})}$ & $1,0878 \mathrm{~kg} \mathrm{~m}^{-2}$ \\
\hline $\mathrm{CV}$ & $33,24 \%$ \\
\hline
\end{tabular}

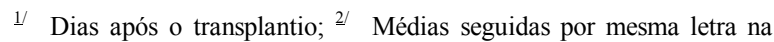
coluna não diferem entre si pelo teste de Tukey $(\mathrm{p}>0,05) ;{ }^{3 /}$ Colheita realizada aos 105 dias após o transplantio.

* Significativo a $5 \%$ pelo teste $\mathrm{F}$; ${ }^{* *}$ significativo a $1 \%$ pelo teste $\mathrm{F}$; ns = não-significativo. 
Na Tabela 7 são apresentadas as porcentagens de bulbos de diferentes classes em relação ao número e peso total da produção de cada período inicial de controle. Os periodos em que o reinício ocorreu aos 56, 70, 84 e 98 DAT promoveram intensas alterações das porcentagens dos bulbos nas diferentes classes de tamanho, tanto para as porcentagens numéricas quanto para peso de bulbos. Desse modo, nos tratamentos que reiniciaram o controle das plantas daninhas após os 42 DAT, houve intensa redução das porcentagens de bulbos das classes 3, 4 e 5 e aumento das porcentagens de bulbos das classes 2 e refugo.

Na Figura 3 estão graficamente representados os valores observados nas curvas de tendências para os dados de peso total da produção de bulbos colhidos nos tratamentos submetidos aos efeitos dos períodos iniciais e de reinício de controle das plantas daninhas na cultura da cebola. Para a análise de regressão em questão, os parâmetros estão apresentados na Tabela 8.

Para os períodos iniciais de controle da comunidade infestante, o modelo mostrou que as primeiras inflexões das curvas ocorreram a partir dos 28 DAT; as reduções foram mais intensas nos tratamentos em que o período de controle inicial foi de 0-0 DAT ou 0-7 DAT. Após este período (28 DAT), cada dia adicional de convivência teve efeito negativo drástico e praticamente linear na produção total de bulbos de cebola - até 70-80 DAT, aproximadamente. Essa é uma fase em que cultura e comunidade infestante apresentam demanda muito mais elevada que a capacidade do meio em fornecer os recursos essenciais ao crescimento das plantas. Até 28 DAT, a demanda não havia suplantado a disponibilidade de recursos e a competição não havia se instalado

Tabela 7 - Distribuição percentual das classes comerciais (CEAGESP), do número e do peso de bulbos de cebola nos grupos de tratamentos de períodos iniciais de controle (0-0, 0-7, 0-14 e 0-21 DAT). FCAV-UNESP, Jaboticabal-SP, 2003

\begin{tabular}{|c|c|c|c|c|c|c|c|c|c|c|c|c|}
\hline \multicolumn{7}{|c|}{ Número de bulbos (\%) } & \multicolumn{6}{|c|}{ Peso de bulbos (\%) } \\
\hline Classe & $28 \mathrm{DAT}^{1 /}$ & 42 DAT & 56 DAT & 70 DAT & 84 DAT & 98 DAT & 28 DAT & 42 DAT & 56 DAT & 70 DAT & $84 \mathrm{DAT}$ & 98 DAT \\
\hline & \multicolumn{12}{|c|}{ 0-0 DAT } \\
\hline refugo & 3,53 & 12,33 & 65,85 & 94,95 & 88,24 & 100,00 & 0,49 & 2,97 & 36,71 & 83,51 & 46,88 & 100,00 \\
\hline 2 & 17,65 & 34,93 & 30,08 & 5,05 & 11,76 & 0,00 & 7,76 & 23,14 & 49,86 & 16,49 & 53,13 & 0,00 \\
\hline 3 & 58,24 & 47,95 & 4,07 & 0,00 & 0,00 & 0,00 & 58,54 & 62,98 & 13,44 & 0,00 & 0,00 & 0,00 \\
\hline 4 & 20,00 & 4,79 & 0,00 & 0,00 & 0,00 & 0,00 & 31,42 & 10,91 & 0,00 & 0,00 & 0,00 & 0,00 \\
\hline \multirow[t]{2}{*}{5} & 0,59 & 0,00 & 0,00 & 0,00 & 0,00 & 0,00 & 1,78 & 0,00 & 0,00 & 0,00 & 0,00 & 0,00 \\
\hline & \multicolumn{12}{|c|}{ 0-7 DAT } \\
\hline refugo & 2,84 & 11,59 & 73,91 & 69,09 & 55,36 & 94,44 & 0,49 & 2,21 & 45,24 & 25,42 & 15,12 & 76,12 \\
\hline 2 & 12,77 & 31,88 & 22,83 & 23,64 & 23,21 & 5,56 & 4,71 & 19,75 & 43,57 & 40,68 & 29,53 & 23,88 \\
\hline 3 & 60,99 & 52,90 & 3,26 & 7,27 & 21,43 & 0,00 & 59,06 & 69,49 & 11,19 & 33,90 & 55,35 & 0,00 \\
\hline 4 & 23,40 & 3,62 & 0,00 & 0,00 & 0,00 & 0,00 & 35,75 & 8,55 & 0,00 & 0,00 & 0,00 & 0,00 \\
\hline \multirow[t]{2}{*}{5} & 0,00 & 0,00 & 0,00 & 0,00 & 0,00 & 0,00 & 0,00 & 0,00 & 0,00 & 0,00 & 0,00 & 0,00 \\
\hline & \multicolumn{12}{|c|}{ 0-14 DAT } \\
\hline refugo & 1,79 & 6,72 & $\begin{array}{l}33,85 \\
33,8 \ldots \ldots\end{array}$ & 74,42 & 66,34 & 100,00 & 0,24 & 0,41 & 12,13 & 48,00 & 33,27 & 100,00 \\
\hline 2 & 14,29 & 26,12 & 50,00 & 23,26 & 27,72 & 0,00 & 6,93 & 14,51 & 55,20 & 45,40 & 48,77 & 0,00 \\
\hline 3 & 55,36 & 45,52 & 16,15 & 2,33 & 5,94 & 0,00 & 51,91 & 47,78 & 32,67 & 6,60 & 17,96 & 0,00 \\
\hline 4 & 28,57 & 21,64 & 0,00 & 0,00 & 0,00 & 0,00 & 40,92 & 37,31 & 0,00 & 0,00 & 0,00 & 0,00 \\
\hline \multirow[t]{2}{*}{5} & 0,00 & 0,00 & 0,00 & 0,00 & 0,00 & 0,00 & 0,00 & 0,00 & 0,00 & 0,00 & 0,00 & 0,00 \\
\hline & \multicolumn{12}{|c|}{ 0-21 DAT } \\
\hline refugo & 0,68 & 4,44 & 35,61 & 69,16 & 89,36 & 85,71 & 0,02 & 0,48 & 12,28 & 37,70 & 65,52 & 59,76 \\
\hline 2 & 19,18 & 15,56 & 40,91 & 25,23 & 10,64 & 14,29 & 9,85 & 8,02 & 40,94 & 40,85 & 34,48 & 40,24 \\
\hline 3 & 57,53 & 61,48 & 21,21 & 5,61 & 0,00 & 0,00 & 53,40 & 62,06 & 38,11 & 21,45 & 0,00 & 0,00 \\
\hline 4 & 21,92 & 18,52 & 2,27 & 0,00 & 0,00 & 0,00 & 35,07 & 29,43 & 8,68 & 0,00 & 0,00 & 0,00 \\
\hline 5 & 0,68 & 0,00 & 0,00 & 0,00 & 0,00 & 0,00 & 1,65 & 0,00 & 0,00 & 0,00 & 0,00 & 0,00 \\
\hline
\end{tabular}

\footnotetext{
${ }^{1 /} \mathrm{DAT}=$ Dias após o transplantio.
} 


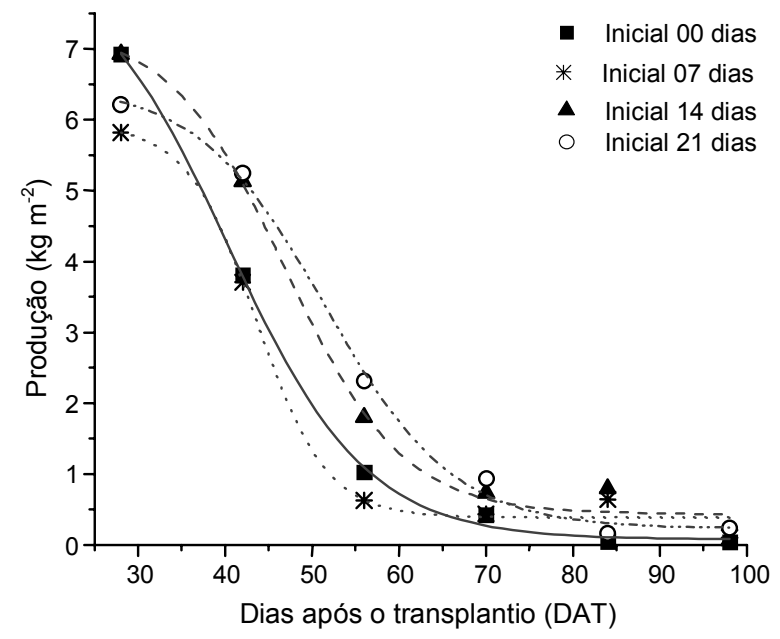

Figura 3 - Efeitos dos períodos iniciais de controle das plantas daninhas estabelecidos em diferentes épocas sobre a produtividade da cultura da cebola 'Mercedes'. FCAV/ UNESP. Jaboticabal-SP. 2003.

Tabela 8 - Parâmetros obtidos pelo modelo de Boltzmann aplicado à densidade de plantas da comunidade infestante com início de desenvolvimento em diferentes épocas após o transplantio da cultura da cebola. FCAV/UNESP. Jaboticabal-SP, 2003

\begin{tabular}{|c|c|c|c|c|c|}
\hline $\begin{array}{c}\text { Período inicial } \\
\text { de controle }\end{array}$ & $\mathrm{R}^{2}$ & $\mathrm{~A} 1$ & $\mathrm{~A} 2$ & $\mathrm{x} 0$ & $\mathrm{dx}$ \\
\hline $0-0$ dias & 0,9991 & 8,3782 & 0,0717 & 40,32 & 7,95 \\
\hline $0-7$ dias & 0,9945 & 5,9246 & 0,3889 & 43,61 & 4,00 \\
\hline $0-14$ dias & 0,9933 & 7,3288 & 0,4339 & 46,96 & 6,72 \\
\hline $0-21$ dias & 0,9970 & 6,5069 & 0,2416 & 51,47 & 7,36 \\
\hline
\end{tabular}

de forma definitiva. Desse modo, as melhores produções foram obtidas quando o periodo inicial de controle foi até 14 DAT e se reiniciou aos 28 DAT até a colheita da cebola.

Considerando que a comunidade infestante que se desenvolveu a partir de diferentes períodos iniciais de controle apresentou grandes diferenças na densidade de indivíduos e, principalmente, de acúmulo de biomassa seca, as diferenças nas inflexões da curva e os modelos sigmoidais de produção não foram relevantes. Esse fato sugere que o final do período em que a cultura pode conviver com as plantas daninhas está mais ligado a uma característica de suscetibilidade da planta nesse período do que à pressão competitiva da comunidade infestante.
Por fim, foi possivel concluir que as principais plantas daninhas da área experimental foram Lycopersicum esculentum, Eragrostis pilosa, Portulaca oleracea e Galinsoga parviflora; não houve interação entre os diferentes períodos de início de controle das plantas daninhas e os períodos em que se reiniciou o controle; a convivência com as plantas daninhas durante os primeiros 98 dias reduziu a produtividade da cebola em $94,5 \%$ e o controle inicial das plantas daninhas deve se prolongar até 14 DAT e ser reiniciado aos 28 DAT, a fim de prevenir reduções significativas na produtividade da cultura da cebola transplantada.

\section{LITERATURA CITADA}

BOND, W; BURSTON, S. Timing the removal of weeds from drilled salad onions to prevent crop losses. Crop Protec., v. 15, n. 2, p. 205-211, 1996.

COMPANHIA DE ENTREPOSTOS E ARMAZÉNS GERAIS DO ESTADO DE SÃO PAULO - CEAGESP. 1999. (Comunicação pessoal)

DEUBER, R.; FORSTER, R. Competição mato x cebola. Campinas: Instituto Agronômico do Estado de São Paulo, 1975. 21 p. (Boletim Técnico, 22)

GRIME, J. P. Estrategias de adapatación de las plantas y procesos que controlan la Vegetación. Mexico, D.F.: Noriega, 1979. p. 79-87.

Van HEEMST, H. D. J. The influence of weed competition on crop yield. Agric. Syst., v. 18, n. 2, p. 81-93, 1985.

MUELLER-DOMBOIS, D.; ELLEMBERG, H. Aims and methods of vegetation ecology. New York: Willey \& Sons, 1974. $547 \mathrm{p}$.

PALLER Jr, E. C. et al. Duration of weed competition and weed control and yield in transplanted onions. Philippine Agric., v. 55, n. 5-6, p. 221-224, 1971.

PINTO-COELHO, R. M. Fundamentos em ecologia. Porto Alegre: Artes Médicas Sul, 2000. p. 87-88.

PITELLI, R. A. Efeitos de períodos de convivência e de controle das plantas daninhas no crescimento, nutrição mineral, e produtividade da cultura da cebola (Allium cepa L.). 1987. 140 f. Tese (Livre Docência em Ecologia) Universidade Estadual Paulista, Jaboticabal, 1987.

PITELLI, R. A. Interferência das plantas daninhas nas culturas agrícolas. Inf. Agropec., v. 11, n. 29, p. 16-27, 1985.

SOARES, D. J. et al. Períodos de interferência das plantas daninhas na cultura da cebola (Allium cepa) transplantada. Planta Daninha, v. 21, n. 3, p. 387-396, 2003.

Planta Daninha, Viçosa-MG, v. 22, n. 4, p. 517-527, 2004 